\title{
Platypnea-orthodeoxia Syndrome Due to Right Ventricular Inflow Tract Obstruction Caused by an Elongated Ascending Aorta: Usefulness of Three-dimensional Cardiac Computed Tomography Imaging in the Sitting Position
}

Yuki Hasegawa ${ }^{1}$, Daisuke Izumi ${ }^{1}$, Yasuhiro Ikami ${ }^{1}$, Takeshi Okubo ${ }^{1}$, Makoto Hoyano ${ }^{1}$, Kazuyuki Ozaki ${ }^{1}$, Noriaki Sato ${ }^{2}$, Takehito Mishima ${ }^{2}$ and Takayuki Inomata ${ }^{1}$

\begin{abstract}
:
An 84-year-old woman presented with dyspnea in the sitting position. Platypnea-orthodeoxia syndrome (POS) was suspected based on arterial desaturation when her posture changed from the supine to the sitting position. Transesophageal echocardiography showed right-to-left shunting enhancement through a patent foramen ovale (PFO) in the sitting position. Three-dimensional (3D) cardiac CT in the sitting position revealed that the elongated ascending aorta compressed the right ventricular inflow tract, resulting in restricted blood flow to the right ventricle and increased right-to-left shunting. This case highlights the role of 3D-CT in the sitting position in the management of POS.
\end{abstract}

Key words: Platypnea-orthodeoxia syndrome, 3-dimensional cardiac computed tomography, patent foramen ovale, elongated aorta, right-to-left shunt

(Intern Med Advance Publication)

(DOI: 10.2169/internalmedicine.8868-21)

\section{Introduction}

Platypnea-orthodeoxia syndrome (POS) is a rare clinical entity that causes hypoxemia in the upright or sitting position (1). It is reportedly caused by right-to-left shunt, which is augmented by anatomical changes in the upright posture (2). A small number of cases of POS have been reported (3-14), and their pathogenesis is complex and not uniform.

We herein report a case in which three-dimensional (3D) cardiac computed tomography (CT) imaging in the sitting position was useful for understanding the detailed pathogenesis of POS.

\section{Case Report}

An 84-year-old woman with lumbar compression fracture was admitted with dyspnea in the sitting position. An elec- trocardiogram was normal, and chest X-ray revealed mild elongation of the upper mediastinum. Transthoracic echocardiography revealed a normal ventricular function without pulmonary hypertension and an atrial septal aneurysm. A blood gas analysis revealed marked hypoxemia with a low partial pressure of arterial oxygen on room air in the sitting position (sitting, $48.1 \mathrm{mmHg}$; supine, $80.1 \mathrm{mmHg}$ ). Accordingly, POS was suspected.

Transesophageal echocardiography (TEE) showed rightto-left shunting through a patent foramen ovale (PFO) in the supine position (Fig. 1A). The shunt flow was enhanced by postural changes from a supine to a sitting position, with a deformity in the PFO structure (Fig. 1B). To confirm the detailed anatomy, cardiac CT (Aquilion ONETM/ViSION FIRST Edition, Canon Medical Systems, otawara, Japan) was performed in the sitting position (with decreased oxygen saturation) and the supine position. A 3D printed model in the supine position showed elongation of the ascending aorta (Fig. 2A, C), which strongly compressed the right ven-

${ }^{1}$ Department of Cardiovascular Medicine, Niigata University Graduate School of Medical and Dental Sciences, Japan and ${ }^{2}$ Division of Thoracic and Cardiovascular Surgery, Niigata University Graduate School of Medical and Dental Sciences, Japan

Received: October 19, 2021; Accepted: November 22, 2021; Advance Publication by J-STAGE: January 13, 2022

Correspondence to Dr. Yuki Hasegawa, y_hase29@yahoo.co.jp 

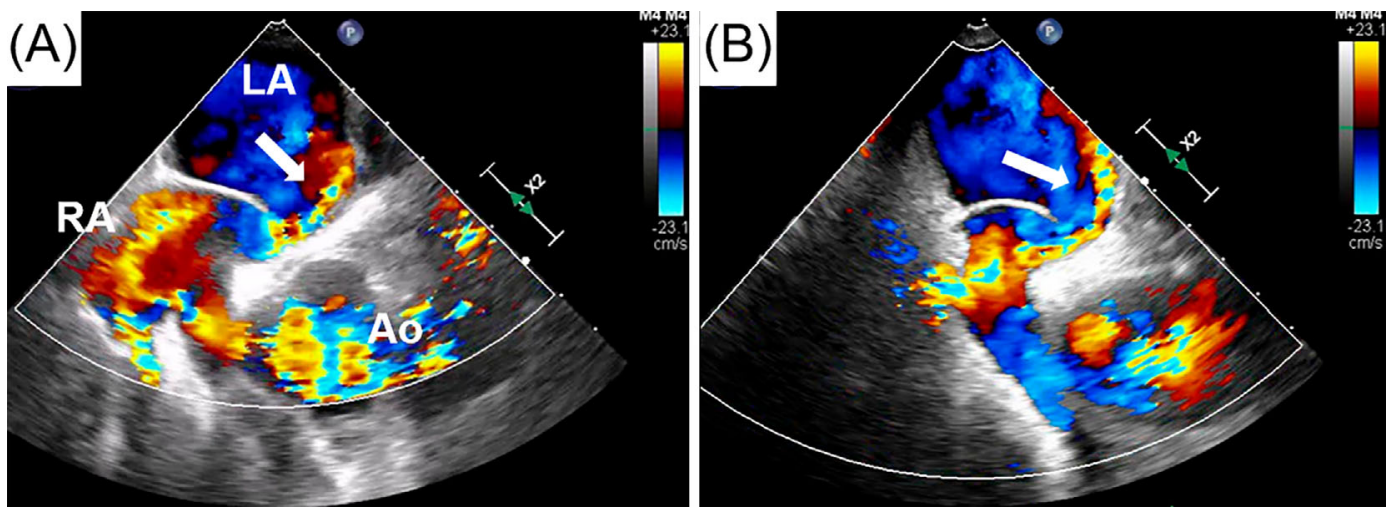

Figure 1. Transesophageal echocardiography with color Doppler recorded with the patient in the supine position (A) and in the sitting position (B). The right-to-left shunt was enhanced with deformity in the structure of the foramen ovale in the sitting position. The arrow indicates the right-to-left shunt flow. Ao: aorta, LA: left atrium, RA: right atrium
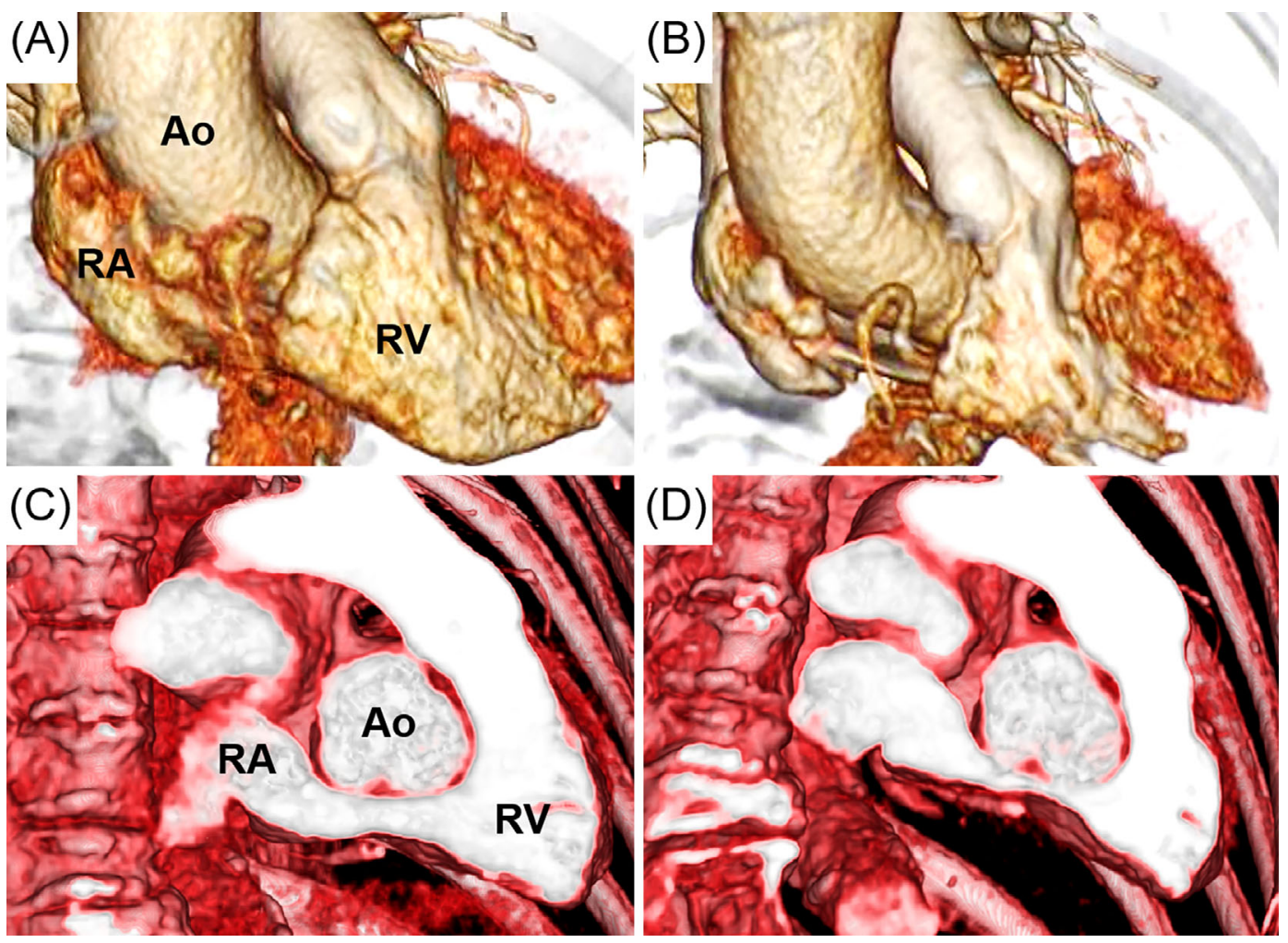

Figure 2. (A, B) Three-dimensional cardiac computed tomography (CT) in the supine position (A) and sitting position (B). The elongated ascending aorta more strongly compressed the right ventricular inflow tract in the sitting position than in the supine position. (C, D) Sagittal section of cardiac CT in the supine position $(C)$ and in the sitting position (D). The elongated ascending aorta caused right ventricular inflow tract obstruction in the sitting position. Ao: aorta, RA: right atrium, RV: right ventricle

tricular inflow tract in the sitting position (Fig. 2B, D).

PFO closure was considered, but we feared this would cause stagnation of blood flow in the right atrium and circulatory. We therefore evaluated the hemodynamics by cardiac catheterization. Conversion from the supine to semisitting position increased the right-to-left shunt rate (23\% to $30 \%$ ) and the mean pressure gradient between right atrium and right ventricle (1 $\mathrm{mmHg}$ to $3 \mathrm{mmHg}$ ). During a PFO balloon occlusion test using a 24-mm diameter AMPLATZER TM Sizing Balloon (St. Jude Medical, St. Paul, MN, USA) in the semisitting position, the oxygen saturation increased from $85 \%$ to $96 \%$ with no hemodynamic changes (no elevation of pressure gradient between right atrium and right ventricle or decrease of systemic blood pressure). 
We performed surgical closure of the PFO without treating the elongated ascending aorta. Subsequently, she no longer experienced hypoxemia in the sitting position. Mild right ventricular inflow tract obstruction in the sitting position remains, but the patient is doing well at two years after surgery.

\section{Discussion}

The proposed pathophysiology of POS involves the coexistence of anatomical components, such as PFO or ASD, and functional components, which enhance right-to-left shunting through postural changes (2). Arterial blood gas measurement in different postures is the initial diagnostic step. Subsequently, right-to-left shunt must be confirmed. TEE is crucial for direct visualization of the shunt flow or structural abnormalities and has been used in some reported cases (4-13).

However, even if POS is diagnosed by these tests, it is challenging to understand its detailed pathogenesis. Previous reports have demonstrated different functional components, including elongation or aneurysm of the ascending aorta (5$7,10,11,13)$, compression by extracardiac structures $(3,14)$, tricuspid regurgitation $(4,9)$, pericardial disease (8), or pulmonary embolism (12). The most common condition associated with POS is PFO with an elongated ascending aorta, but even in these cases, various mechanisms have been reported, including compression of the right atrium $(6,10,13)$, rotation of the entire heart (11), atrial septum deformation $(5,7)$, or changes in the direction of venous return via the inferior vena cava (7). In our case, 3D cardiac CT and TEE suggested that right-to-left shunt enhancement in the sitting position might have been caused by a restricted blood flow to the right ventricle and deformation of the atrial septum by the elongated ascending aorta.

Regarding the treatment of POS, there are cases in which only shunt closure was performed $(6,10)$, or in which the ascending aorta was surgically treated $(7,13)$. In our case, PFO closure was the only surgical treatment because cardiac catheterization confirmed that PFO closure with a balloon did not have a significant hemodynamic impact. Since most POS patients are elderly, low-invasive treatments should be considered. Although percutaneous PFO closure has been reported to be successful in some cases $(5,11)$, trans-catheter technique was not performed in our case because of the strong deformation of the atrial septum by the ascending aorta.

Using multiple modalities may therefore positively contribute to making an accurate diagnosis and the successful treatment of POS. To our knowledge, this is the first report demonstrating right ventricular inflow tract obstruction in
POS, which was identified by $3 \mathrm{D}$ cardiac $\mathrm{CT}$ in the sitting position. This approach was useful for visualizing the pathogenesis.

The authors state that they have no Conflict of Interest (COI).

\section{References}

1. Cheng TO. Platypnea-orthodeoxia syndrome: etiology, differential diagnosis, and management. Catheter Cardiovasc Interv 47: 64-66, 1999.

2. Agrawal A, Palkar A, Talwar A. The multiple dimensions of Platypnea-Orthodeoxia syndrome: A review. Respir Med 129: 3138, 2017.

3. Alkhouli M, Gagel A, Mathur M, O’Murchu B. Platypneaorthodeoxia syndrome: an unusual complication of partial liver resection. Intern Med 54: 1067-1069, 2015.

4. Borgaonkar SP, Lam WW, Razavi M, Parekh DR. PlatypneaOrthodeoxia Syndrome Caused by an Intracardiac Shunt. Tex Heart Inst J 47: 298-301, 2020.

5. Chopard R, Meneveau N. Right-to-left atrial shunting associated with aortic root aneurysm: a case report of a rare cause of platypnea-orthodeoxia syndrome. Heart Lung Circ 22: 71-75, 2013.

6. Harada K, Nakagawa K, Ohtsuka H, et al. Platypnea-orthodeoxia Syndrome Induced by Multiple Vertebral Compression Fractures and an Atrial Septal Defect. Intern Med 57: 971-973, 2018.

7. Hasegawa M, Nagai T, Murakami T, Ikari Y. Platypnoeaorthodeoxia syndrome due to deformation of the patent foramen ovale caused by a dilated ascending aorta: a case report. Eur Heart J Case Rep 4: 1-4, 2020.

8. Hashimoto M, Okawa Y, Baba H, Nishimura Y, Aoki M. Platypnea-orthodeoxia syndrome combined with constrictive pericarditis after coronary artery bypass surgery. J Thorac Cardiovasc Surg 132: 1225-1226, 2006.

9. Hatani Y, Tanaka H, Kajiura A, et al. Sudden Onset of PlatypneaOrthodeoxia Syndrome Caused by Traumatic Tricuspid Regurgitation With Ruptured Chordae Tendineae After Blunt Chest Trauma. Can J Cardiol 34: 1088.e11-1088.e13, 2018.

10. Kazawa S, Enomoto T, Suzuki N, et al. Platypnea-orthodeoxia Syndrome in a Patient with an Atrial Septal Defect: The Diagnosis and Choice of Treatment. Intern Med 56: 169-173, 2017.

11. Meier B, Lock JE. Contemporary management of patent foramen ovale. Circulation 107: 5-9, 2003.

12. Salvetti M, Zotti D, Bazza A, et al. Platypnea and orthodeoxia in a patient with pulmonary embolism. Am J Emerg Med 31: 760.e1e2, 2013.

13. Takashima N, Suzuki T, Asai T, Hosoba S. Successful surgical repair of platypnea-orthodeoxia syndrome in a patient with cerebral infarction. Interact Cardiovasc Thorac Surg 15: 178-180, 2012.

14. Vallurupalli S, Lodha A, Kupfer Y, Tessler S. PlatypneaOrthodeoxia syndrome after repair of a paraesophageal hernia. BMJ Case Rep 2013: 2013.

The Internal Medicine is an Open Access journal distributed under the Creative Commons Attribution-NonCommercial-NoDerivatives 4.0 International License. To view the details of this license, please visit (https://creativecommons.org/licenses/ by-nc-nd/4.0/).

(C) The Japanese Society of Internal Medicine Intern Med Advance Publication 